Virginia Commonwealth University

VCU Scholars Compass

2012

\title{
Temperature-dependent Kelvin probe measurements of band bending in p-type GaN
}

\section{Foussekis}

Virginia Commonwealth University

J.D. McNamara

Virginia Commonwealth University, mcnamarajd@vcu.edu

\section{A. A. Baski}

Virginia Commonwealth University, aabaski@vcu.edu

Michael A. Reshchikov

Virginia Commonwealth University,mreshchi@vcu.edu

Follow this and additional works at: http://scholarscompass.vcu.edu/phys_pubs

Part of the Physics Commons

Foussekis, M., McNamara, J. D., Baski, A. A., et al. Temperature-dependent Kelvin probe measurements of band bending inp-type GaN. Applied Physics Letters, 101, 082104 (2012). Copyright (C) 2012 AIP Publishing LLC.

\section{Downloaded from}

http://scholarscompass.vcu.edu/phys_pubs/26

This Article is brought to you for free and open access by the Dept. of Physics at VCU Scholars Compass. It has been accepted for inclusion in Physics Publications by an authorized administrator of VCU Scholars Compass. For more information, please contact libcompass@vcu.edu. 


\title{
Temperature-dependent Kelvin probe measurements of band bending in p-type GaN
}

\author{
M. Foussekis, J. D. McNamara, A. A. Baski, and M. A. Reshchikov ${ }^{\text {a) }}$ \\ Department of Physics, Virginia Commonwealth University, Richmond, Virginia 23284, USA
}

(Received 10 May 2012; accepted 6 August 2012; published online 21 August 2012)

\begin{abstract}
The band bending in a Mg-doped, $p$-type GaN film grown by hydride vapor phase epitaxy was studied at various temperatures. At $295 \mathrm{~K}$, the band bending in dark was calculated to be approximately $-1.5 \mathrm{eV}$. However, when the sample was heated to $600 \mathrm{~K}$ for $1 \mathrm{~h}$ in dark before performing a measurement at $295 \mathrm{~K}$, the calculated value of band bending in dark became about $-2.0 \mathrm{eV}$. These results are explained by the fact that increasing the sample temperature exponentially increases the rate at which the band bending restores and allows for a more accurate value of band bending to be measured. (C) 2012 American Institute of Physics. [http://dx.doi.org/10.1063/1.4747203]
\end{abstract}

Characterizing the surface of GaN films is of paramount importance, since surface conditions influence device performance. It is known that the surfaces of $n$ - and p-type GaN commonly exhibit upward and downward band bending as a result of excess negative or positive surface charge, respectively. The exact source of this charge is still unknown. ${ }^{1}$ The band bending and related depletion region have detrimental effects on the optical and electrical properties of GaN-based devices. ${ }^{2}$ We consider two potential sources of surface charge: internal and external. Possible internal sources of surface charge include semiconductor surface states due to dangling bonds, reconstruction, structural and point defects, spontaneous polarization, or impurities. External sources of surface charge can include a surface oxide layer or adsorbed species. It has been established that a monolayer of chemisorbed oxygen exists on the surface of $\mathrm{GaN}$, which may form a thin $(\sim 1 \mathrm{~nm})$ surface oxide. ${ }^{3}$ The values of the upward band bending reported for $n$-type $\mathrm{GaN}$ are approximately $1 \mathrm{eV}$ and do not vary much between reports. ${ }^{4-7}$ However, the reported values of band bending for $p$-type $\mathrm{GaN}$ vary widely from about -0.8 to $-3.0 \mathrm{eV} .^{8-13}$ The reason for such a wide range of band bending values in $p$-type $\mathrm{GaN}$ has, to date, not been given much consideration.

In the literature, there are few studies in which the sample temperature was varied while performing x-ray photoelectron spectroscopy (XPS) or ultraviolet photoelectron spectroscopy (UPS) measurements. The peak positions from XPS and UPS can be used to measure the near-surface band bending. However, the drawback is that the incoming X-rays generate a surface photovoltage (SPV) and reduce the band bending. ${ }^{13}$ We believe that the temperature at which the measurements were taken affects the measured values of band bending calculated from these reports. Tracy et al. ${ }^{8}$ measured the band bending in $p$-type GaN using XPS and UPS. In these measurements, the sample was annealed at $1140 \mathrm{~K}$ for $15 \mathrm{~min}$ and then, the temperature was reduced to $295 \mathrm{~K}$ before taking data. The calculated downward band bending in their sample was about $-0.8 \mathrm{eV}$.

\footnotetext{
${ }^{\text {a) }}$ Author to whom correspondence should be addressed. Electronic mail: mreshchi@vcu.edu.
}

To minimize the effect of the SPV in XPS measurements, it is necessary to increase the sample temperature. Long and Bermudez ${ }^{13}$ performed a temperature-dependent study of $p$-type GaN using XPS and UPS. Although the authors did not explicitly estimate the band bending, it can be calculated using the surface Fermi-level position and free carrier concentration. When the temperature was increased from 300 to $700 \mathrm{~K}$, the magnitude of the calculated band bending increased from 0.77 to $2.15 \mathrm{eV}$ for $p$-type $\mathrm{GaN}$ which can be attributed, at least partially, to the reduction of the SPV magnitude with increasing temperature. We have estimated that a typical $\mathrm{x}$-ray intensity ${ }^{13}$ on the order of $10^{14} \mathrm{~cm}^{-2} \mathrm{~s}^{-1}$ would produce an SPV signal of at least $0.2 \mathrm{eV}$. In comparison to XPS, Kelvin probe measurements of the surface potential are expected to be more reliable, since these measurements in dark do not affect the measured value of band bending. From surface potential data and the concentration of free holes reported by Eyckeler et al., ${ }^{12}$ we calculated the band bending in their $p$-type GaN sample to be about $-3.0 \mathrm{eV}$. In their experiment, the sample was heated to about $1100 \mathrm{~K}$ in dark, before measurements with a Kelvin probe were performed at $150 \mathrm{~K}$.

In this work, we studied the effect of temperature on $\mathrm{SPV}$ in $p$-type GaN in an effort to explain the disparity of band bending values reported in the literature. The SPV technique can be used to estimate the band bending in semiconductors. Illumination with band-to-band light generates electron-hole pairs in the depletion region that are separated due to the strong electric field. In $p$-type materials, band-toband illumination causes photo-generated electrons to accumulate at the surface which reduces the net positive charge and therefore decreases the band bending. We have previously shown that the surface potential can be measured by the contactless Kelvin probe method, and that the band bending can then be estimated from SPV measurements and the dark contact potential difference (CPD) signal. ${ }^{14-16}$

We investigated several $p$-type GaN samples grown by hydride vapor phase epitaxy (HVPE), metal-organic vapor phase deposition (MOCVD), and molecular beam epitaxy (MBE). The sample which was studied extensively is a Mg-doped GaN film, with a thickness of about $3 \mu \mathrm{m}$, which was grown on $c$-plane sapphire by HVPE at TDI, Inc. ${ }^{17}$ The 
concentration of uncompensated shallow acceptors $\left(N_{A}-\right.$ $N_{D}$ ) for this sample was estimated from capacitance-voltage measurements to be about $6.5 \times 10^{18} \mathrm{~cm}^{-3}$. Ohmic contacts were formed by depositing Ni/Au layers around the sample periphery. The Hall effect data were ambiguous, most likely due to the existence of a thin, degenerate $n$-type layer near the GaN/sapphire interface. ${ }^{18}$ However, the sample exhibited $p$-type conductivity from SPV and hot probe measurements. Assuming a compensation ratio of 0.5 , we estimate that $N_{A}=1.3 \times 10^{19} \mathrm{~cm}^{-3}$ and $N_{D}=6.5 \times 10^{18} \mathrm{~cm}^{-3}$, which provides a concentration of free holes of $p=7 \times 10^{15} \mathrm{~cm}^{-3}$ at $295 \mathrm{~K}$, if the ionization energy of the acceptor $E_{A}=0.18 \mathrm{eV}$. To account for errors in these estimates, we additionally analyzed the dependence of the Fermi-level position on the compensation ratio, which was varied from 0.2 to 0.8 . At most, the Fermi-level position changed by $\pm 0.04 \mathrm{eV}$ within the range of temperatures used in this work and, therefore, does not introduce any significant error in our estimates of band bending.

The surface potential was measured using a Kelvin probe mounted inside an optical cryostat with a base pressure of about $10^{-6}$ mbar. Prior to illumination, the sample was allowed to restore in two different ways: stored in dark for at least 1 day at $295 \mathrm{~K}$ or heated to $600 \mathrm{~K}$ for $1 \mathrm{~h}$. In both cases, the sample was illuminated from the front side using a $\mathrm{HeCd}$ laser $(325 \mathrm{~nm})$. The laser beam was passed through a window in the cryostat, and then through a perforated $4 \mathrm{~mm}$ diameter probe before reaching the sample surface. The maximum light intensity incident on the sample surface was $\sim 40 \mathrm{~mW} / \mathrm{cm}^{2}$ and could be attenuated by about 10 orders of magnitude with a series of neutral density filters.

To estimate the near-surface band bending in dark, $\Phi_{0}$, the SPV behavior can be fit using a thermionic model. ${ }^{14}$ The SPV value, $y_{0}$, generated at a given excitation intensity, $P_{0}$, can be used to estimate $\Phi_{0}$ in $p$-type GaN with the relation

$$
y_{0}=-\eta k T \ln \left(\frac{c P_{0}}{R_{0}}+1\right)
$$

with

$$
R_{0}=s_{p} N_{V} \exp \left(\frac{\Phi_{0}+\left(E_{V}-F\right)}{\eta k T}\right)
$$

where $\eta$ is an ideality factor (expected to be close to one), $k$ is Boltzmann's constant, $T$ is the temperature, $c$ is a geometrical factor that accounts for the portion of photons that are absorbed in the depletion region, $R_{0}$ is the rate at which holes move from the bulk to surface per unit time per unit area, $s_{p}$ is the surface recombination velocity for holes, $N_{v}$ is the effective density of states in the valence band, and $E_{v}$ and $F$ are the positions of the valence band maximum and Fermi level, respectively. Because the value of $c P_{0} / R_{0}$ in Eq. (1) is much greater than one for the range of light intensities used in this study, Eqs. (1) and (2) simplify to

$$
y_{0} \approx \Phi_{0}-\left(F-E_{v}\right)+\eta k T \ln \left(\frac{s_{p} N_{v}}{c P_{0}}\right) .
$$

This expression can be used to find $\Phi_{0}$ if the other parameters are known. In our experiment, $P_{0}$ was calculated from the laser power density, and the parameter $c$ was estimated to be 0.13 for front-side illumination of $\mathrm{GaN}$ with a calculated depletion region width of $12 \mathrm{~nm}$. The value of $\left(F-E_{v}\right)$ was estimated to vary from 0.20 to $0.23 \mathrm{eV}$ with increasing temperature from 295 to $650 \mathrm{~K}$ for the studied HVPE-grown $\mathrm{GaN}$ sample. We assumed that $s_{p}$ for $\mathrm{GaN}$ lies within the range of $10^{3}-10^{7} \mathrm{~cm} / \mathrm{s}$ with the most probable value of $10^{5} \mathrm{~cm} / \mathrm{s} .{ }^{19}$ Note that changing the value of $s_{p}$ by four orders of magnitude introduces an error in the estimate of band bending of only $\pm 0.2 \mathrm{eV}$. Finally, the temperature dependence of $N_{v}$ has a negligible effect on $y_{0}$ because of its logarithmic dependence. Thus, it is expected from Eq. (3) that the magnitude of the SPV should decrease almost linearly with increasing temperature.

To examine the effect of sample temperature on the SPV generated in $p$-type GaN, the surface was illuminated for $1 \mathrm{~s}$ with $P_{0}=7 \times 10^{16} \mathrm{~cm}^{-2} \mathrm{~s}^{-1}$ at temperatures from 295 to $500 \mathrm{~K}$. The SPV as a function of sample temperature is shown in Fig. 1, for the case when the sample was not preheated (empty circles) before the measurement, and for the case when it was pre-heated at $600 \mathrm{~K}$ in dark for $1 \mathrm{~h}$ before the measurement (filled circles). Note that at $295 \mathrm{~K}$, the absolute value of the SPV for the sample with pre-heating is significantly larger than for the sample without pre-heating (see arrow in Fig. 1). Interestingly, when the sample was not preheated, the magnitude of the SPV increased from 0.55 to $0.8 \mathrm{eV}$ as the temperature increased from 295 to $500 \mathrm{~K}$. This behavior contradicts the thermionic model [Eq. (3)] which predicts that the magnitude of the SPV should decrease with increasing sample temperature. However, when the same sample was pre-heated at $600 \mathrm{~K}$ for $1 \mathrm{~h}$ before performing a measurement, the magnitude of the SPV decreased from 1.2 to $0.8 \mathrm{eV}$ as the temperature increased from 295 to $500 \mathrm{~K}$. Such a large value of SPV $(-1.2 \mathrm{eV})$ observed after preheating indicates that the magnitude of $\Phi_{0}$ apparently exceeds $1.2 \mathrm{eV}$ at $295 \mathrm{~K}$, whereas the estimated lower bound of $\left|\Phi_{0}\right|$ for the sample without preheating is only $0.55 \mathrm{eV}$.

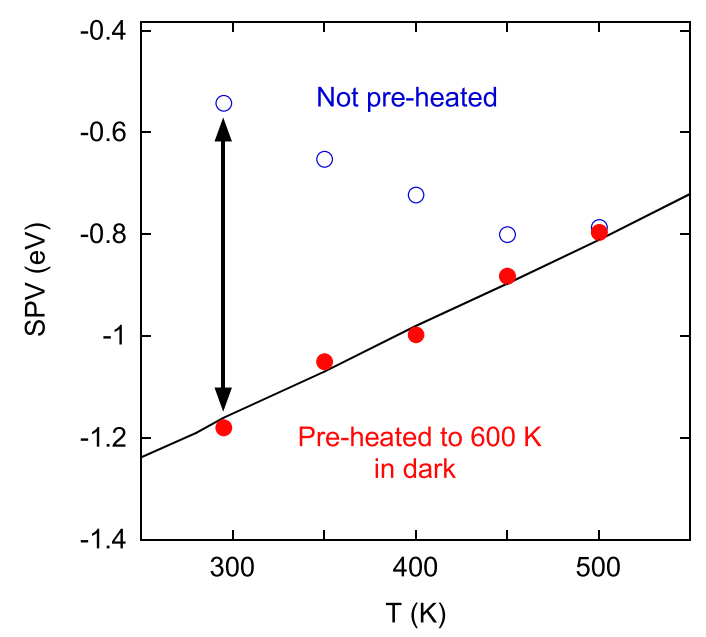

FIG. 1. SPV generated with $P_{0}=7 \times 10^{16} \mathrm{~cm}^{-2} \mathrm{~s}^{-1}$ as a function of sample temperature. Sample was pre-heated in dark at $600 \mathrm{~K}$ for $1 \mathrm{~h}$ (filled circles) or was not pre-heated (empty circles). The solid line is calculated using Eq. (3) with the following parameters: $c=0.13, \eta=1, \Phi_{0}=-1.45 \mathrm{eV}$, and $s_{p}=10^{5} \mathrm{~cm} / \mathrm{s}$. To find the temperature dependence of $F$ in Eq. (3), we used the following parameters: $N_{A}=1.3 \times 10^{19} \mathrm{~cm}^{-3}, N_{D}=6.5 \times 10^{18} \mathrm{~cm}^{-3}$, and $E_{A}=0.18 \mathrm{eV}$. 
The process of pre-heating the sample at high temperatures increases the rate of restoration for the band bending in dark, thereby providing more accurate values for subsequent SPV measurements. Therefore, the sample temperature is important when estimating the band bending in dark.

In order to estimate $\Phi_{0}$, we fit the data in Fig. 1 by Eq. (3) with $\eta=1$ (solid line), where $\Phi_{0}$ is the only fitting parameter. With this method, the band bending in dark for the $p$-type $\mathrm{GaN}$ sample, after being pre-heated, is found to be $-1.45 \mathrm{eV}$. We consider the magnitude of $1.45 \mathrm{eV}$ as the lower bound for $\left|\Phi_{0}\right|$ because $\eta$ varies between 1.3 and 3.1 in p-type GaN, according to the excitation intensity and temperature dependencies to be discussed later. These large values of $\eta$ greatly affect the value of $\Phi_{0}$ obtained from Eq. (3). Conversely, the value of $\eta$ for $n$-type $\mathrm{GaN}$ is equal to one for a wide range of temperatures, and $\Phi_{0}$ can be reliably and accurately determined from SPV measurements. ${ }^{20}$ However, $\eta$ is much larger than one for $p$-type $\mathrm{GaN}$, and the above estimates may not be accurate and should be regarded with caution. An alternative method to estimate the band bending using Kelvin probe measurements is described below.

The band bending in dark can also be determined from the dark CPD signal using the expression

$$
\Phi_{0}=\phi_{M}-\chi-\left(E_{g}+E_{V}-F\right)-q V_{C P D},
$$

where $\chi$ is the electron affinity for $\mathrm{GaN}(3.2 \mathrm{eV}),{ }^{21} E_{\mathrm{g}}$ is the band gap width $(3.43 \mathrm{eV}$ at $295 \mathrm{~K})$, and $q V_{C P D}$ is the measured CPD multiplied by the electron charge. The work function of the stainless steel probe, $\phi_{M}$, was estimated to be $4.8 \mathrm{eV}$ from the measurement of a gold foil and $4.45 \mathrm{eV}$ from data for an $n$-type GaN sample with $\Phi_{0}=1.05 \mathrm{eV} .{ }^{20}$ The latter was considered as a more reliable value, because the band bending in this sample was accurately established from the SPV measurements by using the thermionic model. Figure 2 shows the values of band bending determined from CPD data for several samples at temperatures between 295 and $500 \mathrm{~K}$. These measurements were taken after the samples were pre-heated at $600 \mathrm{~K}$ which allowed the band bending to

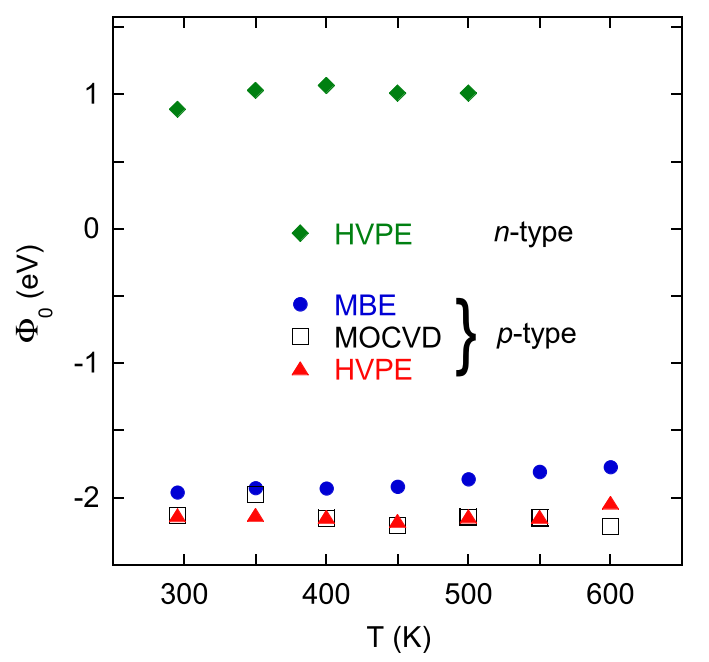

FIG. 2. Band bending in dark for both $n$ - and $p$-type GaN calculated from CPD measurements in dark using Eq. (4) for $p$-type and using Eq. (31) from Ref. 14 for $n$-type. The data were obtained after pre-heating the sample for $1 \mathrm{~h}$ at $600 \mathrm{~K}$ in dark before each measurement. restore more fully. In addition to the HVPE sample, the data for two other $p$-type GaN samples grown by MBE and MOCVD and an $n$-type GaN sample grown by HVPE are shown for comparison. The CPD signal for all of these samples was obtained in a similar manner. The calculated band bending for all samples is independent of temperature and equals about $+1 \mathrm{eV}$ for the $n$-type sample and about $-2 \mathrm{eV}$ for all $p$-type GaN samples. Without preheating the sample, the calculated value of $\Phi_{0}$ for the $n$-type GaN sample does not change, whereas for the $p$-type samples, the calculated absolute value of the band bending was typically smaller by about $0.3 \mathrm{eV}$ than the values from experiments with preheating at $600 \mathrm{~K}$. Note that the preheating can in principle affect the work function of the probe, and other errors in the parameters may propagate in the calculation. While the agreement between the results obtained from the SPV and CPD data is qualitative rather than quantitative for $p$-type GaN, both methods indicate that the large band bending cannot fully restore in dark at room temperature and, consequently, is underestimated.

To further investigate how temperature affects the dark value of band bending, the restoration of the SPV after illumination was measured at temperatures from 295 to $500 \mathrm{~K}$. After illumination of the sample has ceased, the band bending is expected to restore to its dark value with a rate proportional to the sample temperature. The restorations of the SPV after short (1 s) UV illuminations at different temperatures are shown in Fig. 3. The amount of time required for the band bending to fully restore in dark has a strong dependence on temperature and can be fit by a thermionic model $^{14}$

$$
y(t) \approx y_{0}+\eta k T \ln \left(1+\frac{t}{\tau}\right)
$$

with

$$
\tau=\frac{\eta k T}{R_{0}} \frac{p_{s}(0)}{2 \Phi_{0}} \exp \left(\frac{y_{0}}{\eta k T}\right)
$$

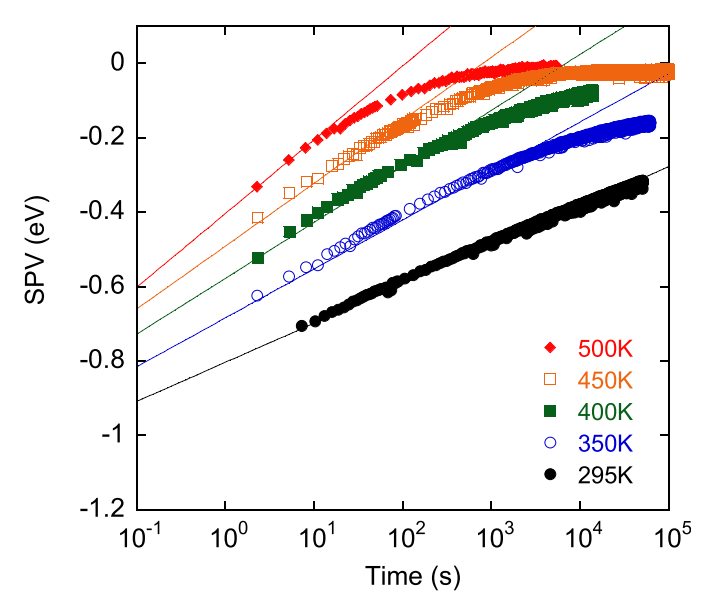

FIG. 3. Restorations of SPV after short (1 s) UV illuminations at $P_{0}=7 \times 10^{16} \mathrm{~cm}^{-2} \mathrm{~s}^{-1}$ for temperatures from 295 to $500 \mathrm{~K}$. Each measurement is taken after preheating the sample at $600 \mathrm{~K}$ for $1 \mathrm{~h}$. The fits are calculated using Eq. (5) with $\tau=0.001 \mathrm{~s}$ and $y_{0}=-1.12,-1.08,-1.03,-1.0$, and $-0.9 \mathrm{eV} ; \eta=1.8,1.9,1.9,1.9$, and 2 ; for $T=295,350,400,450$, and $500 \mathrm{~K}$, respectively. 


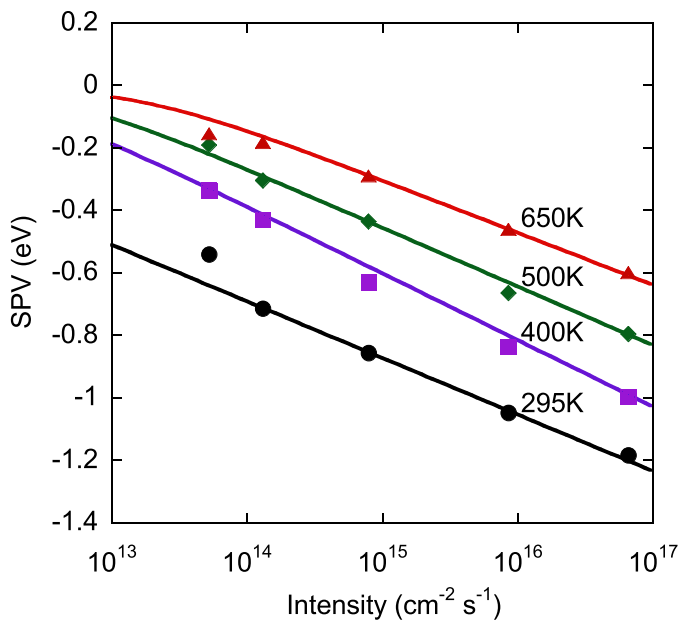

FIG. 4. Intensity-dependent SPV data taken at the indicated temperatures after preheating at $600 \mathrm{~K}$ for $1 \mathrm{~h}$ in dark. The solid lines are calculated using Eq. (1) with parameters: $c=0.13 ; \eta=3.1,2.7,1.9$, and $1.3 ; R_{0}=2 \times 10^{9}$, $2 \times 10^{11}, 5 \times 10^{11}$, and $2 \times 10^{12} \mathrm{~cm}^{-2} \mathrm{~s}^{-1}$ for $T=295,400,500,650 \mathrm{~K}$, respectively.

where $y_{0}$ is the SPV when illumination has ceased, $t$ is the time elapsed after illumination, $\tau$ is a characteristic time delay which exponentially depends on the near-surface barrier, and $p_{s}(0)$ is the net density of positively charged states at the surface. Equation (5) is a simplified version of the restoration equation from a previous work that does not account for the complete restoration of band bending. ${ }^{14}$ This equation is only meant to predict the behavior at the beginning of restoration and the slope. For this reason, there is no asymptotic behavior in the fits when the SPV data show complete restoration. As expected, the fits to the SPV data using Eq. (5) indicate that the initial slope of the restoration is faster at higher temperatures. By extrapolating the fit for $295 \mathrm{~K}$, the SPV is predicted to fully restore in approximately $10^{8} \mathrm{~s}$ (a few years). This takes four orders of magnitude more time than the complete restoration of the band bending at $500 \mathrm{~K}$, which should occur in about $10^{4} \mathrm{~s}(3 \mathrm{~h})$. From these restoration rates, it is clear that heating the sample in darkness allows band bending values larger than $1 \mathrm{eV}$ to fully restore, thereby providing more accurate baselines for SPV and CPD measurements.

Figure 4 shows the SPV as a function of excitation intensity at various temperatures for the HVPE $p$-type GaN sample after pre-heating. As predicted by Eq. (3), the magnitude of the SPV at any given excitation intensity decreases with increasing temperature, and the magnitude of the SPV increases logarithmically with excitation intensity at all temperatures. However, the slope of the SPV signal versus illumination intensity for temperatures from 295 to $650 \mathrm{~K}$ is larger than expected: $\eta$ ranges from 3.1 to 1.3 , respectively. The slope of the SPV dependence on light intensity begins to approach the predicted value at high temperatures, with $\eta=1.3$ at $650 \mathrm{~K}$. The dependence of $\eta$ on $T$ may indicate that the high resistivity of our $p$-type GaN sample is affecting the Kelvin probe SPV measurements. At higher temperatures, the sample conductivity greatly increases, and any effect due to the high resistivity of the sample should decrease. The larger than expected slope at low temperatures may be related to the Dember potential which arises due to the different mobilities of electrons and holes in GaN. ${ }^{1}$ These measurements also indicate that the band bending values obtained at higher temperatures are more reliable.

In conclusion, we have observed that the temperature at which $p$-type GaN samples are kept in dark can, at least partially, explain the wide range of band bending values reported in the literature. Measurements performed at higher temperatures allow for the band bending to more fully restore and therefore provide larger and more accurate values of band bending in dark. If the measurements are performed at $295 \mathrm{~K}$ without pre-heating, however, then the band bending will not have sufficient time to fully restore and a "practical" value of band bending in dark of about $-1.5 \mathrm{eV}$ will be measured. It appears that the actual downward band bending is about $-2.0 \mathrm{eV}$. This value was calculated from the dark CPD signal and is in agreement with the values estimated from fitting the SPV data with a thermionic model.

This work was supported by the National Science Foundation (DMR-080679). The authors are grateful to A. Usikov (TDI Inc.), to R. Calarco (Germany), and D. Koleske (Sandia National Labs) for providing HVPE, MBE, and MOCVD $\mathrm{GaN}$ samples, respectively, and the Morkoç group at VCU for fabricating the sample contacts.

${ }^{1}$ L. Kronik and Y. Shapira, Surf. Sci. Rep. 37, 1 (1999).

${ }^{2}$ S. Nakamura, IEICE Trans. Electron. E83-C, 529 (2000).

${ }^{3}$ T. Sasaki and T. Matsuoka, J. Appl. Phys. 64, 4531 (1988).

${ }^{4}$ V. M. Bermudez, J. Appl. Phys. 80, 1190 (1996).

${ }^{5}$ G. Koley and M. G. Spencer, J. Appl. Phys. 90, 337 (2001).

${ }^{6}$ C. I. Wu, A. Kahn, N. Taskar, D. Dorman, and D. Gallagher, J. Appl. Phys. 83, 4249 (1998).

${ }^{7}$ S. Sabuktagin, M. A. Reshchikov, D. K. Johnstone, and H. Morkoç, Mat. Res. Soc. Symp. Proc. 798, Y5.39 (2003).

${ }^{8}$ K. M. Tracy, W. J. Mecouch, R. F. Davis, and R. J. Nemanich, J. Appl. Phys. 94, 3163 (2003).

${ }^{9}$ M. Alonso, R. Cimino, and K. Horn, Phys. Rev. Lett. 64, 1947 (1990).

${ }^{10}$ H. Sezen, E. Ozbay, O. Aktas, and S. Suzer, Appl. Phys. Lett. 98, 111901 (2011).

${ }^{11}$ S. Barbet, R. Aubry, M. A. di Forte-Poisson, J. C. Jacquet, D. Deresmes, T. Mélin, and D. Théron, Appl. Phys. Lett. 93, 212107 (2008).

${ }^{12}$ M. Eyckeler, W. Monch, T. U. Kampen, R. Dimitrov, O. Ambacher, and M. Stutzmann, J. Vac. Sci. Technol. B 16, 2224 (1998).

${ }^{13}$ J. P. Long and V. M. Bermudez, Phys. Rev. B 66, 121308 (2002).

${ }^{14}$ M. A. Reshchikov, M. Foussekis, and A. A. Baski, J. Appl. Phys. 107, 113535 (2010).

${ }^{15}$ M. Foussekis, A. A. Baski, and M. A. Reshchikov, Appl. Phys. Lett. 94, 162116 (2009).

${ }^{16}$ M. Foussekis, M. A. Reshchikov, and A. A. Baski, J. Vac. Sci. Technol. B 29, 041205 (2011).

${ }^{17}$ Now Ostendo Technologies, Inc., 6185 Paseo del Norte, Ste 200, Carlsbad, CA 92011, Ostendotech.com.

${ }^{18}$ D. C. Look, C. E. Stutz, R. J. Molnar, K. Saarinen, and Z. Liliental-Weber, Solid State Commun. 117, 571 (2001).

${ }^{19}$ P. Ščajev, K. Jarašiūnas, S. Okur, Ü. Özgür, and H. Morkoç, J. Appl. Phys. 111, 023702 (2012).

${ }^{20}$ J. D. McNamara, M. Foussekis, H. Liu, H. Morkoç, M. A. Reshchikov, and A. A. Baski, Proc. SPIE 8262, 826213 (2012).

${ }^{21}$ H. Nienhaus, M. Schneider, S. P. Grabowski, W. Monch, R. Dimitrov, O. Ambacher, and M. Stutzmann, Mater. Res. Soc. Symp. Proc. 680, E4.5 (2001). 